Association for Information Systems

AIS Electronic Library (AISeL)

\title{
On Your Mark, Ready, Search: A Framework for Structuring Literature Search Strategies in Information Systems
}

\author{
Thorsten Schoormann \\ Universität Hildesheim \\ Dennis Behrens \\ Universität Hildesheim \\ Michael Fellmann \\ Universität Rostock \\ Ralf Knackstedt \\ Universität Hildesheim
}

Schoormann, Thorsten; Behrens, Dennis; Fellmann, Michael; and Knackstedt, Ralf, "On Your Mark, Ready, Search: A Framework for Structuring Literature Search Strategies in Information Systems" (2021). Wirtschaftsinformatik 2021 Proceedings. 3.

https://aisel.aisnet.org/wi2021/ZMethods/Track01/3

This material is brought to you by the Wirtschaftsinformatik at AIS Electronic Library (AISeL). It has been accepted for inclusion in Wirtschaftsinformatik 2021 Proceedings by an authorized administrator of AIS Electronic Library (AISeL). For more information, please contact elibrary@aisnet.org. 


\title{
On Your Mark, Ready, Search: \\ A Framework for Structuring Literature Search Strategies in Information Systems
}

\author{
Thorsten Schoormann ${ }^{1}$, Dennis Behrens ${ }^{1}$, Michael Fellmann ${ }^{2}$, and Ralf Knackstedt ${ }^{1}$ \\ ${ }^{1}$ University of Hildesheim, Information Systems, Hildesheim, Germany \\ \{thorsten.schoormann,ralf.knackstedt\}@uni-hildesheim.de; dennisb.mail@gmx.de \\ ${ }^{2}$ University of Rostock, Business Information Systems, Rostock, Germany \\ \{michael.fellmann\}@uni-rostock.de
}

\begin{abstract}
Researchers often face challenges already in the early stages of a literature review, and thus, struggle in getting started with the search and in organizing the process. This starting point is however of great relevance because design decisions such as in terms of corpus creation have impacts on the entire results of the review. By following the design science paradigm, we present the 'Search Canvas', a generic framework that aims at supporting the (creative) process of exploring, specifying, and visually representing a literature search strategy. In doing this, we contribute to the understanding of what components need to be considered when deriving a search strategy and provide an instrument that enables researchers to iteratively plan and communicate such strategies.
\end{abstract}

Keywords: Literature Search Strategy, Keyword-based Search, Canvas-based Framework, Literature Review, Design Science.

\section{Introduction and Problem Awareness}

The relevance of conducting rigorous literature reviews in any kind of project is uncontroversial (e.g., [1-6]). Reviewing the literature is a crucial starting point of every research, and thus, used by researchers at different professional stages from students to graduates to become familiar with or deepen their view on a phenomenon [7, 8]. In contrast to narrative reviews, systematic reviews seek to ensure rigorousness through well-defined processes as well as quality criteria such as traceability, systematicity, or reproducibility $[2,9,10]$. Ensuring rigorousness enables other researchers to fully decode the review's path as well as to build on and complement the review's findings.

Even though available methodological guidance (e.g., in the form of quality criteria or procedure models) helps to perform literature reviews, it is however still challenging to get started with the review and to formulate an appropriate search strategyespecially for students and novice researchers. We argue that this can be attributed to three main indications: First, although the review's starting point is of great importance because "[failures] have a considerable impact on the resulting review's quality" [11], (IS) researchers often face the 'dilemma at the start' [11] because they have no clear 
orientation where to begin searching like in other disciplines that have, for instance, established overviews of reference databases [12]. Second, although IS research is often intertwined or linked with adjacent disciplines, conventional corpus creation often focuses on parts of a phenomenon without the benefit of a larger context, which leads to deficits in terms of identifying the majority of research [13]. Third, several authors stated dangers in terms of a review's linearity, particularly with regards to forcing reviewers to strictly apply 'methodological checklists' and not allowing them to adapt search strategies based on an emerging understanding [14,17]. Methodological flexibility is however demanded, for instance, to make the review's research questions more precise in successive stages [15], develop a search phrase across iterations [16], and to perform an (initial) planning phase not only at a review's beginning [14].

In addition, from our personal experience as researchers, lecturers, and authors, we also observed this discrepancy between the (particularly novice) researcher's expectations and the systematic literature review's degree of difficulty. On the one hand, novices tend to prefer performing systematic reviews because they seem to have an easy to follow set of specified start activities from selecting keywords to summarizing the results in the form of a concept matrix for example. On the other hand, however, asking the researcher about details concerning the decisions they have made, it often becomes evident that there are serious deficits in the review's rigorousness (e.g., search query was changed at a late stage). To bridge these gaps, we aim at deriving a framework that facilitates the (creative) process of specifying a literature search strategy, and thus, helps to divide and conquer the complex task of getting started with a review. Therefore, we raise the following research question (RQ): How to design a tool that supports specifying and communicating literature search strategies?

In attempting to answer this, we carried out a design science research (DSR) study in which we iteratively build and evaluate a framework, the 'Search Canvas', which synthesizes the basic components that need to be considered during a literature search. As canvas-based tools facilitate exploration and ideation [18], brainstorming, and collaboration across disciplinary boundaries [19], we believe that this might be a helpful tool to overcome challenges with regard to starting a search as well. Since we adapt known solutions in the form of the canvas (e.g., known from the business model canvas [20]) to new problems, we position our work as exaptation research (i.e., "adopt solutions from other fields" [21]). Our contribution seeks to complement available knowledge on how to conduct literature reviews and especially help novices organizing the starting point of a review, for instance, by enabling them to jointly ideate within a team of reviewers, communicate ideas to collect feedback from experts, and document (interim) results. In doing this, we also hope to respond to calls for better documentation of design decisions in IS research, which is a basis for transparency [5].

\section{Research Background: Searching for Literature}

A well-documented specification of the search strategy contributes to the rigorousness of a literature review because it ensures transparency, systematicity, traceability, clarity, and reproducibility $[5,10,14,22,23]$. Generally, searching for literature can be 
carried out through (1) a keyword-based procedure or (2) a systematic personal reading [8]. Both have pros and cons: Personal reading needs a lot of time and keyword-based searches depend on the selection of proper items. The identification of keywords in particular is challenging [2] as certain concepts might have "dozens of completely different words or expressions for designating the same phenomenon" [8]. Hence, the process of planning and conducting a search is highly iterative and requires continuously finding/validating terms [24].

In order to handle the complexity of the search phase, authors have provided methodological recommendations for literature reviews. For example, [25] suggested phases including conceptualization of topic and literature search, and argued that a review should start with a broad conception of what is known about a certain topic to provide definitions of key terms (e.g., by using seminal books). The search itself should contain information related to databases selected and keywords used. [26] proposed guidelines consisting of steps including searching for the literature in which the authors claimed for explicit descriptions of the search process and the justification of how the comprehensiveness can be assured (e.g., journal selection and clear keyword definition). In [27], a procedure model is presented comprising generating a search strategy that consists of using various combinations of keywords, drawing up a list of synonyms and alternative spellings, as well as deriving search strings by means of Boolean operators. The author called for explicitly documenting and justifying the entire search. By laying a focus on transparency and systematicity, [14] proposed beginning with developing a review plan (e.g., formulate a research question, describe review goals, publish review protocol), searching the literature (e.g., define criteria for inclusion, select databases, describe search strategy), and selecting studies (e.g., describe screening process, list included studies). [13] explored the creation of a corpus and differentiated between steps for identifying boundaries and construct a corpus.

In attempting to operationalize such recommendations, so-called review protocols are provided that help to structure and document literature searches. Although review protocols are usually employed for systematic reviews, they are according to [11] valuable for all types of reviews. Those protocols aim at minimizing the bias in the study and are a critical element within a review [28]. Especially in the context of software engineering, progress towards a unified protocol has been made: Several authors [27, 29] deduced a search process template differentiating between electronic databases (name, search for each database, date of search, years), journal hand search (name, years), conference proceedings, efforts to identify unpublished studies (e.g., researchers contacted), and other sources (date, URL); [30] specified elements for the search process including search strings, inclusion criteria, exclusion criteria, initial primary study selection, and final primary study selection; [28] complemented these ideas through a procedure-perspective and defined the first phase for a review with steps for specifying a research question, develop a review protocol, and validate it. As another example, [31] presented search planning worksheets containing elements for databases, grey literature, journals, and experts to contact. In the IS discipline, [11] emphasized the great relevance of such protocols and [26] argued that protocols are particularly worthwhile to have in case of multiple reviewers within a single project to ensure clearness and consistency in terms of the search procedure. 
Nevertheless, although recommendations related to the search strategy are available, still various challenges occur - for novice researchers in particular — such as identifying adequate items or combining items into a search phrase [2, 8, 11]. Additionally, as there is a heterogeneous set of protocols, there is a need for a holistic overview of relevant components in IS. In contrast to subsequent phases in the review such as categorizing literature and managing bibliographic data that is well-supported by tools [22], the creation of a search strategy is still more an 'art than sciences' that requires a welldefined structure and a significant amount of knowledge and experience.

\section{Research Design: A Design Science Study}

\subsection{Justification and Overview of Design}

In order to achieve our overall goal, we developed the Search Canvas. We decided to derive a canvas for literature searches because of four main reasons: First, visualization in general helps to structure information and allows us to overcome cognitive overloads [32]. Second, so-called visual inquiry tools such as the business model canvas [20] support the joint process of exploration and ideation, are expected to be effective in individual and group settings, and are easy to use [18]. Third, some of the canvasses have experienced worldwide success and great acceptance (e.g., business model canvas has been downloaded more than 5.000.000 times) [19]. Based on a survey, users stated that by means of canvas they achieved, for instance, better conversation, a shared understanding, better brainstorming, better collaboration across disciplines because it is intuitive, visual, and simple [19]. Moreover, [33] identified positive effects on the actual learning and the perceived ease of learning. Fourth, canvasses have been adapted to methodological purposes such as design science [34] as well as in domains as diverse as data-driven businesses [35], service design [36], or sustainability [37]. We believe that these reasons and benefits are also helpful for literature searches, especially to (1) address the iterative and creative character of specifying a search strategy, (2) communicate interim strategies and enable other stakeholders and experts to provide feedback, and (3) enable interdisciplinary teams of researchers to collaborate with the canvas as a so-called boundary object (i.e., provide interfaces for different worlds [38]).

Our overall study is structured by utilizing Hevner et al.'s [39] framework (see Figure 1). This framework bridges the environment and the knowledge base with the actual DSR project. For building our artifact, the Search Canvas, we adapted principles for visual inquiry tools [26] and relied on different sources: Conceptualization of available methodological guidance for literature reviews to draw on existing knowledge; a systematic review of articles published in high-ranked IS journals to examine how the search strategy is reported/created in 'practice'; think aloudsessions - as part of an established research paradigm investigating people's cognitive process and behavior [40] - to reveal how researches actually derive a search strategy and which problems occur. For evaluation, we aimed at gathering both qualitative insights utilizing a survey of potential users and quantitative insights through performing an $\mathrm{A} / \mathrm{B}$-test that helps to disclose the effects of applying our artifact. 

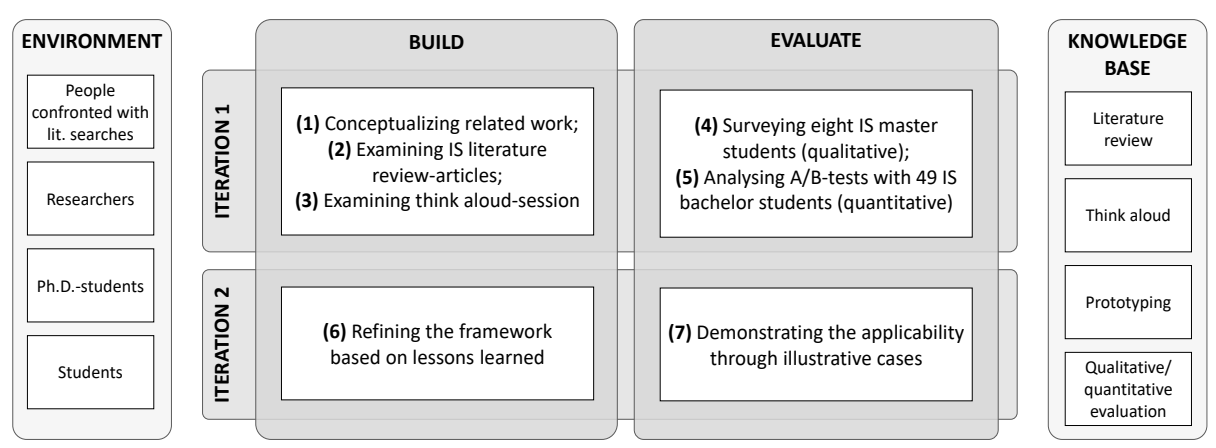

Figure 1. Research design including different steps (1-7), according to [39]

In total, we ran through two major iterations (i.e., design cycles) of building and evaluating our Search Canvas. Next, both iterations are described in detail.

\subsection{Iteration 1 - Initial Search Canvas}

Conceptualizing related work (1). First, we aimed to build on available knowledge, and thus synthesized methodological guidelines and components that need to be considered during the literature search phase by means of a rather narrative review approach [4]. In doing so, we deduced relevant concepts from methodological work on literature reviews (see also Section 2).

Examining literature review-articles (2). Afterward, we analyzed how existing studies in IS research describe their search. ${ }^{1}$ Therefore, we conducted a broad search with the following search phrase: "literature review" OR "literature analysis". Since the first popular articles in IS research regarding systematic literature reviews start in early 2000, we limited the results to 'from 2000'. As sources, we selected leading IS journals from the AIS Senior Scholars 'Basket. As a result of the rather broad search phrase, we found 999 articles. Following [3], a complete keyword search as well as an evaluation of titles and abstracts was applied. The articles that did not comprise related terms such as 'literature review' or 'literature analysis' were eliminated. The remaining articles $(n=135)$ were verified by the full text, and non-relevant articles (i.e., the methodology is not described explicitly) were eliminated $(n=51)$.

We examined the obtained sample according to the following characteristics: 'Search items specified' (are the items defined in a fix or a fuzzy manner); 'no. of items' (the number of specified keywords); 'derivation of search items' (description and justification of why the items are selected); 'search sources' (sources selected in a fix or fuzzy manner); 'search phrase' (logically combination of the items). As an excerpt from the results ${ }^{2}$, we found a total of 51 studies with 209 search items and 7 search phrases. Hence, there is an average of approximately 4.09 search items per paper. Nonetheless, the amount of search items and sources that were selected within a review varies considerably. As an example, whereas [41] used 21 search items, [42] only one

1 The entire list of IS articles from the review is available upon request.

2 For more details on the results of our literature review, see also [16]. 
item. Moreover, we found that $27.45 \%$ defined their keywords in a fuzzy manner (e.g., with "such as") and only $13.72 \%$ described their phrases, which makes it hard to trace the process in detail. Although important to ensure rigorousness, only $54.90 \%$ followed a research methodology of which 18 articles refer to Webster and Watson [3].

Examining think aloud-sessions (3). To disclose people's cognitive processes [44], we carried out several think alouds [43]. Following the ' $10 \pm 2$ rule' for evaluations [45], we selected eight participants: Six Ph.D.-students (IS, Information Management, and Operation Research) from one to four years of work experiences; two master students (IS). Thus, our selection comprises especially novice and early-stage researchers. The participants came from different universities, and all of them conducted at least one literature review by themselves. All sessions were carried out individually in a separate office with three actors (participant, moderator, and observer). For collecting data, an audio recording was made and observations were protocolled. The workshop was divided into three major parts: First, in the introduction, we welcomed the participants, described the rules of the workshop, outlined the task that has to be executed. Second, the participants received a sheet that summarizes important information related to think aloud and the task to be carried out. The task aims at creating a search phrase for a literature review (here, on 'sustainability and business models'). If a participant stopped talking, the moderator reminds her/him to continue talking. In case that a certain part of a search strategy or at least the entire strategy was comprehensible, the moderator forwarded it to the next step. Third, in the final part, the participants were asked to answer some questions regarding literature reviews in general. In line with our study's purpose, two researchers coded the obtained data independently to determine (a) problems and challenges while setting up a search strategy as well as (b) typical activities that were performed during the specification of a search strategy.

Surveying master students (4). To get qualitative (in-depth) feedback on the artifact's applicability and usefulness from potential users, we provided the initial Search Canvas and a short questionnaire to eight IS master-leveled students enrolled in a course in which they had to conduct a literature review (i.e., incentive in the form of a grade). Therefore, the students employed the Search Canvas and answered questions as well as gave general feedback in terms of the canvas's usefulness (see Section 4).

Analyzing A/B-tests (5). Additionally, to obtain quantitative insights and to explore which differences occur when applying the Search Canvas against not-applying it, we performed an A/B-test with 49 bachelor students enrolled in IS (incentive: bonus points for the exam at the end of the course). In doing this, the artifact is investigated in a controlled environment with potential 'real users' and 'real tasks' (here: to create a search strategy). 24 students completed the task with the help of the Search Canvas and 25 students completed the task without using the Search Canvas (see Section 4).

\subsection{Iteration 2 - Refined Search Canvas}

Refining the framework (6). Based on the lessons learned from the evaluation episode in Iteration 1, we refined (i.e., built) the Search Canvas (see Section 4 for refinements).

Demonstrating the applicability (7). Following Peffers et al. [46], illustrative scenarios that apply "an artifact to a synthetic or real-world situation aimed at 
illustrating suitability or utility of the artifact" are among the most frequently used approaches for evaluating frameworks. This is further emphasized by [39] who stated that "detailed scenarios around the artifact [should be constructed] to demonstrate its utility". In line with this, we selected two different published IS literature reviewarticles and apply the refined Search Canvas to (re-)document the literature search performed by the authors (see Section 5).

\section{Iteration 1 - Initial Search Canvas}

\subsection{Building the Initial Search Canvas}

Drawing on the findings derived in steps $1-3$, we built our Search Canvas. Initially, the canvas differentiates between the following components ${ }^{3}$ : Purpose of search, related domains, seminal work, search item candidates, search items, search sources, combination candidates, final search phrase, and project management (see Figure 2).

\begin{tabular}{|l|l|l|l|}
\hline \multicolumn{2}{|l|}{ Purpose of Search } \\
\hline \multicolumn{2}{|l|}{ Related Domains } \\
\hline Seminal Work & Search Items & $\begin{array}{l}\text { Combination } \\
\text { Candidates }\end{array}$ & $\begin{array}{l}\text { Final } \\
\text { Search Phrase }\end{array}$ \\
\hline $\begin{array}{l}\text { Search Item } \\
\text { Candidates }\end{array}$ & Search Sources & \\
\hline Project Management & & \\
\hline
\end{tabular}

Figure 2. Our initial framework for search strategies (Search Canvas, Iteration 1)

\subsection{Surveying Master Students (Qual. Evaluation of Initial Search Canvas)}

In step 4, to get feedback on the artifact's usefulness from potential users (e.g., novice researchers), we provided the canvas as well as a short questionnaire to eight IS masterleveled students with (a) closed questions that should be answered through a Likertscale and (b) open questions that should be answered through text fields. By analyzing the closed questions, two observations emerged (see Table 2): First, documentation is the highest-ranked purpose of the Search Canvas (5.0). Second, the overall helpfulness of the Search Canvas is quite high (4.62). In terms of open questions, we obtained positive feedback referring to the framework's ability to structure the search process (P2, P6, P7, P8), the framework's simplicity (P5), and the support of brainstorming keywords (P5). In contrast, one improvement could be deduced: Ambiguous difference between 'search item candidates' and 'search items' (P5). In Iteration 2, we have merged and renamed these components (see Section 5).

3 For space reasons, we described the components of the refined Search Canvas—see Section 5. 
Table 1. Questionnaire results (Likert-scale from 1 low to 7 high)

\begin{tabular}{|c|c|c|c|c|c|c|c|c|c|}
\hline & $P 1$ & $P 2$ & $P 3$ & $P 4$ & $P 5$ & $P 6$ & $P 7$ & $P 8$ & $A V G$ \\
\hline I am familiar with lit. reviews. & 6 & 4 & 1 & 2 & 4 & 4 & 5 & 6 & 4 \\
\hline $\begin{array}{l}\text { I am familiar with procedure } \\
\text { models for literature reviews. }\end{array}$ & 6 & 3 & 1 & 2 & 4 & 4 & 4 & 5 & 3.62 \\
\hline $\begin{array}{l}\text { I am familiar with tools for lit. } \\
\text { reviews. }\end{array}$ & 5 & 3 & 1 & 2 & 5 & 4 & 5 & 6 & 3.87 \\
\hline I think the framework is helpful. & 6 & 5 & 5 & 3 & 4 & 4 & 5 & 5 & 4.62 \\
\hline $\begin{array}{l}\text { Documentation of the search is } \\
\text { easier via the framework. }\end{array}$ & 6 & 6 & - & 4 & 4 & 4 & 4 & 6 & 5 \\
\hline $\begin{array}{l}\text { Specification of the search strategy } \\
\text { is easier via the framework. }\end{array}$ & 7 & 3 & - & 4 & 6 & 4 & 5 & 6 & 4.83 \\
\hline
\end{tabular}

\subsection{Analyzing A/B-tests (Quant. Evaluation of Initial Search Canvas)}

Step 5 sought to gather insights about potential effects occurring from the usage of the framework. Therefore, we conducted a preliminary A/B-test that distinguishes between two groups (i.e., experiment and control group) each with a set of randomly assigned students. The test was held within an obligatory course for (IS) research methods. The participation was anonymous and voluntary, and thus, there was no direct incentive in the form of a grade/credits but participating helped the students to successfully pass the overall course. The task ( 30 minutes) that had to be solved was formulated as follows: 'Systematically create a search strategy (i.e., the first phases of a literature review in particular) that seeks to identify articles providing extensions for the process modeling notation BPMN 2.0'. All of the participants were familiar with at least one procedure model for literature reviews (e.g., [25] was discussed within the course) and with process modeling (part of the curriculum). A total of 49 IS bachelor students participated in the test, 24 students in Group A (i.e., with the Search Canvas and a short description) and 25 students in Group B (i.e., without the Search Canvas). The results of the demographic and experience data are summarized in Table 2.

Table 2. Demographics/experiences (scale: from 1 low to 5 high; group averages reported)

\begin{tabular}{lcccc}
\hline & Group A & Group B & Difference & Median \\
\hline I am familiar with BPMN (domain of review). & 2.78 & 3.18 & 0.40 & 2.98 \\
I am familiar with lit. reviews. & 2.83 & 2.82 & 0.01 & 2.83 \\
I am familiar with the procedure model [25]. & 1.67 & 1.27 & 0.39 & 1.47 \\
I am familiar with additional procedure models & 2.61 & 2.59 & 0.02 & 2.6 \\
for lit. reviews. & & & & \\
\hline
\end{tabular}

Table 3 reports (a) the frequency in percent (\%) of how often a typical search strategy component is addressed by the groups (e.g., how often did participants refer to 'related disciplines') as well as (b) the number of items within a component (e.g., how many 'seminal works' are specified, accumulated across the answers). As this evaluation task was executed individually, the component 'project management' has not been used. 
Table 3. Results from the A/B-test (Group A = with canvas; Group B = without canvas)

\begin{tabular}{lcccc}
\hline \multirow{2}{*}{ Components } & \multicolumn{2}{c}{ Group A $(\mathbf{n = 2 4})$} & \multicolumn{2}{c}{ Group B $(\mathbf{n = 2 5})$} \\
\cline { 2 - 5 } & $\begin{array}{c}\text { \% addressed } \\
\text { component }\end{array}$ & $\begin{array}{c}\text { No. of } \\
\text { elements }\end{array}$ & $\begin{array}{c}\text { \% addressed } \\
\text { component }\end{array}$ & $\begin{array}{c}\text { No. of } \\
\text { elements }\end{array}$ \\
\hline Purpose of search & $55 \%$ & -- & $88 \%$ & -- \\
Related disciplines & $50 \%$ & 6 & $0 \%$ & 0 \\
Seminal works & $33 \%$ & 8 & $11 \%$ & 1 \\
Search item collection & $61 \%$ & 46 & $22 \%$ & 7 \\
Search sources & $50 \%$ & 22 & $44 \%$ & 4 \\
Preliminary combination & $5 \%$ & 1 & $0 \%$ & 0 \\
Final search phrase & $0 \%$ & -- & $33 \%$ & -- \\
Project management & n.a. & n.a. & n.a. & n.a. \\
\hline
\end{tabular}

Based on the results, five main observations emerged: First, the group with the canvas has addressed more components of a literature search (e.g., 33\% specified 'seminal work' in contrast to $11 \%$ from the non-canvas group; $61 \%$ specified 'search items' in contrast to $22 \%$ from the non-canvas group). Second, the component 'related disciples' is only addressed by the canvas-group. Third, in addition to these advantages, the results indicate that the 'purpose of search' is described more frequently in the non-canvas group (88\% against 55\%). Fourth, two components were addressed rather low, namely 'preliminary combinations' ( $5 \%$ with canvas against $0 \%)$ and 'search phrases' $(0 \%$ with canvas against 33\%) - this might be due to the given task during the think aloudsessions that focuses primarily on the first steps of creating a search strategy. Fifth, even though the component 'search source' is addressed equally $(50 \%$ with canvas against $44 \%$ ), the number of potential search items is considerably higher within the canvas-group (46 items against 7 items).

Furthermore, we could observe that the component 'related domains' often contains websites, so the term 'domain' was misunderstood. To overcome this shortcoming, we revised the wording in Iteration 2 (see Section 5).

\section{Iteration 2 - Refined Search Canvas}

\subsection{Refining the 'Search Canvas'}

Based on the lessons learned from Iteration 1, three major refinements have been made (step 6): First, we have merged 'search item candidates' and 'search items' to 'search item collection' because participants mostly did not use both components. Second, we have renamed 'related domains' into 'related disciplines' because some participants tended to provide websites. Third, we have renamed 'combination candidates' into 'preliminary combinations' and have added more guiding questions for the usage. Moreover, we decided to rename the component 'project management' into 'collaboration' as this is quite broad and users might think of controlling, etc., which is not meant. Overall, in line with the $7 \pm 2$ heuristic [47] - the magical number that 
describes the number of chunks handled through the short-term memory-, the refined canvas comprises nine distinct but interrelated components (see Figure 3).

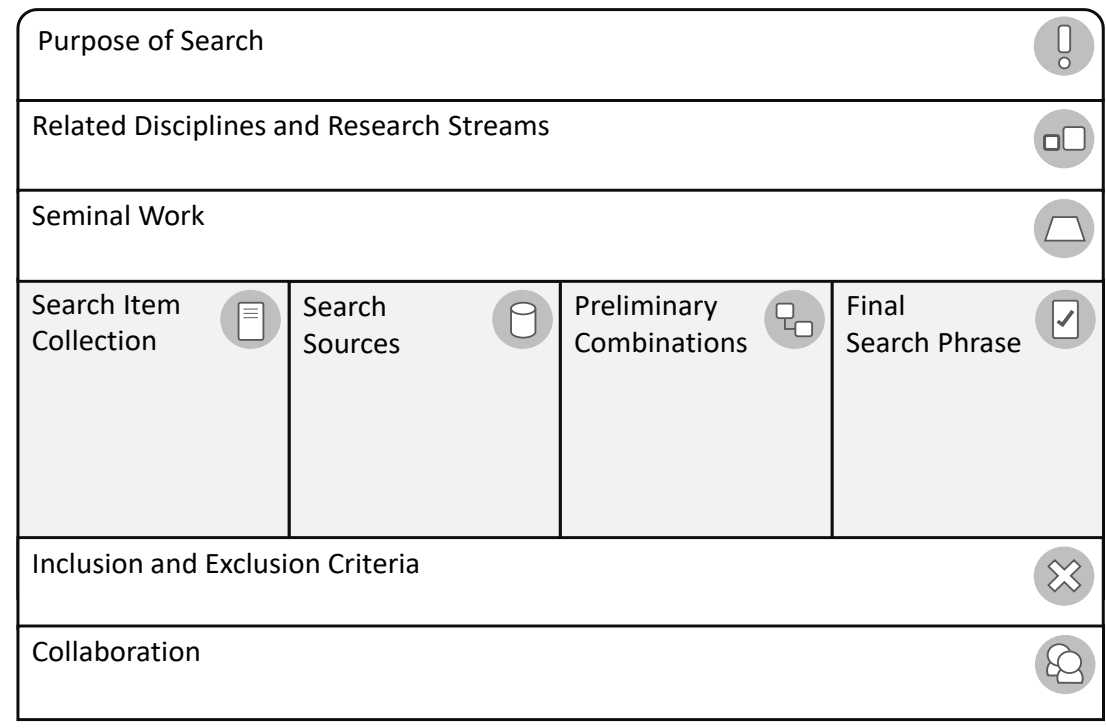

Figure 3. Framework for literature search strategies (Search Canvas, Iteration 2)

In the following section, the components of the Search Canvas are described in more detail. To do so, we refer to the three sources examined during the artifact building (steps 1-3) as well as to selected evaluation episodes (steps 4-5).

Purpose of search. The structure of the review to be conducted highly depends on the overall goal and the research questions that are formulated within a project [11]. Thus, the review's key questions $[14,27,28]$ and purposes should be clearly stated [22, 26] as well as the actual problem that is addressed by means of the review [9].

Related disciplines and research streams ${ }^{* 4}$. Describing the specific researcher's perspective on the phenomenon of interest is helpful because the readers might more easily follow why specific decisions are made during the review. [13] pointed out that reviews often see only a part of the phenomenon for which reason it would benefit from the larger context. Particularly in emerging fields in IS such as digital transformation or artificial intelligence, phenomena become increasingly interdisciplinary. Furthermore, by determining interfaces to other disciplines and research streams, potential search terms and sources can be deduced.

Seminal work. The participants of the think aloud-sessions emphasized that they usually start with a review by exploring seminal authors and co-authors, seminal publications, and seminal books from a certain phenomenon of interest. This is further evident by methodological literature, for instance, "a preliminary search for seminal publications can help researchers to identify and select search terms" [11] and identification of relevant literature can be grounded on 'main authors' [24].

4 Component titles that are marked with an * have been refined or added during Iteration 2. 
Search item collection*. The identification of potential keywords is a crucial and iterative part of the search process. During the think alouds, we observed different strategies for identifying possible search items: Looking up translations, synonyms, related words in dictionaries, as well as modifications of a term like the base form of a certain word (e.g., lemmatization). After identifying potential keywords (mostly in the form of a 'candidate list'), suitable ones were selected. To foster this process, creativity techniques such as brainstorming might be worthwhile to apply. This is also supported by methodological guidance that, for instance, recommend engaging with a topic through encyclopedias, textbooks, edited books, and working definitions $[11,26]$ as well as exploring synonyms, abbreviations, and alternative spellings [27, 31].

Search sources. The selection of appropriate sources is highlighted through plenty of statements. For example, [22] argued for selecting suitable databases, publication outlets, and citation indexing services. [9, 15, 26, 29] named potential electronic sources including Google Scholar, ProQuest, Scopus, EBSCO, IEEE Xplore, ACM Digital Library, Elsevier, and the AIS Electronic Library. [27] clustered potential sources into journals, grey literature such as reports, and conference proceedings. [13] listed possible sources including books, book chapters, journal articles, conference articles, monographs, and unpublished manuscripts.

Preliminary combinations*. To combine selected search items researchers should (a) consider Boolean operators and additional logical operators that are implemented by a search engine or dataset used for the review $[1,28,31]$ as well as (b) verify various alternatives of combining the search items [27]. During the think aloud-sessions, participants also combined their search items by applying logical connectors such as AND, OR, and NOT as well as by using specific search expressions such as "--" for the exclusion of items, or "**" as a placeholder for unknown words/endings (truncation).

Final search phrase. Since some participants argued that "[..] the hardest step in a search is actually putting together the keywords", we decided to split this step into two components for potential candidates and the final search phrase. Doing this, we hope to foster creativity by suggesting a separate first step that should allow an easy collection of combinations of terms (i.e., divergent thinking). Afterward, in a second step, the user can select appropriate combinations (i.e., convergent thinking). Available recommendations on literature reviews support this, for instance: [11] stated that most literature reviews in IS research are conducted through a search phrase, which helps to determine the review's scope and objectives, [22] argued for specifying a search strategy with a precise set of search terms used, and [14] emphasized to report the 'full electronic search strategy' to contribute to the review's transparency.

Inclusion and exclusion criteria*. During the qualitative evaluation (step 4), we could obtain feedback in terms of the importance of appropriate approaches that help to filter the sample to be collected. Therefore, we added a component for inclusion and exclusion criteria, which is also supported by methodological guidance, especially for scoping reviews [4]. In more detail, [27] specified inclusion criteria based on a specific time span (e.g., 01/2004-06/2007), [27] excluded articles that did not define a research question, and [1] suggest aligning such criteria along with the research question.

Collaboration. Often research is not an isolated endeavor, so that different researchers collaboratively work on a certain project-in particular in highly 
interdisciplinary fields. Since there is an increasing amount of available publications that need to be reviewed, a team of researchers is required to handle this amount [26], which needs to be coordinated [28], for instance, during the screening steps [4]. It might also be helpful to consult (domain) experts that, for example, verify or suggest a set of relevant keywords or relevant search sources [31].

\subsection{Exemplary Guiding Questions}

In addition, to guide the application of the framework, we propose exemplary key questions for each of the components (see Table 4).

Table 4. Exemplary key questions for guiding the framework's usage

\begin{tabular}{ll}
\hline \multicolumn{1}{c}{ Component } & \multicolumn{1}{c}{ Exemplary key questions } \\
\hline $\begin{array}{l}\text { Purpose of } \\
\text { search }\end{array}$ & What is the overall goal of the study? \\
\hline $\begin{array}{l}\text { Related } \\
\text { disciplines }\end{array}$ & Which are related disciplines of the phenomenon of interest? \\
\hline $\begin{array}{l}\text { Seminal } \\
\text { work }\end{array}$ & Which streams of research/genres exist on the phenomenon? articles, books, etc. exist? \\
\hline $\begin{array}{l}\text { Search item } \\
\text { collection }\end{array}$ & Which seminal authors and co-authors exist? \\
\hline $\begin{array}{l}\text { Search } \\
\text { sources }\end{array}$ & Which abbreviations and alternative spellings exist? \\
\hline $\begin{array}{l}\text { Preliminary } \\
\text { combinations }\end{array}$ & Which databases and citation indexing services can be used? \\
\hline $\begin{array}{l}\text { Final search } \\
\text { phrase }\end{array}$ & Which (syntax) expressions are supported by a search source? \\
\hline $\begin{array}{l}\text { Inclusion and } \\
\text { exclusion }\end{array}$ & Which combinations would have the most promising results? \\
criteria & When to include or exclude a paper to be reviewed? \\
\hline $\begin{array}{l}\text { Collaboration } \\
\text { Dherch to be reviewed seem to be relevant for the review? }\end{array}$ & Who is your research collaborator and what is his/her role? \\
\hline
\end{tabular}

\subsection{Demonstrating the Applicability}

According to [46], illustrative scenarios are among the most frequently used approaches for evaluating frameworks. Following this, in step 7, we selected two IS studies from our sample of literature review-articles and described them through our Search Canvas (Figure 4): First, [48] who sought to identify structuring themes within 20 years of IS-enabled organizational transformation (purpose), which was inspired by Scott Morton in 1991 (seminal work). At the intersection of strategic management and IT-enabled business transformation (related disciplines), the authors lay an IS-view on that phenomenon. Thus, they searched in IS outlets such as EJIS and JIT (sources) by employed several keywords including 'strategic transformation' (search items), which 
were combined utilizing an OR-operator (phrase). During the search, [48] particularly included empirical studies and excluded studies linking transformation to enterprise systems, etc. (inclusion/exclusion). Second, [49] examined IS studies employing Grounded Theory to disclose the specific role of axial coding (purpose). The topic, placed within the field of qualitative research methods (related streams), is wellgrounded on previous work such as from Strauss \& Corbin and Glaser (seminal work). Hence, [49] searched for the keywords 'strauss', 'corbin', and 'axial' (search items) in the AIS Senior Scholar's Basket (source), which were combined through a Boolean OR (search phrase). The authors excluded articles that do not refer to the specified seminal authors or do not employ axial coding (inclusion/exclusion). Both studies are conducted by several authors (collaboration).

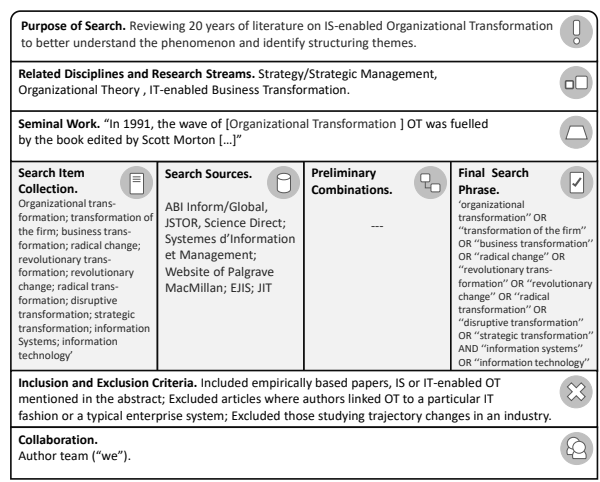

Based on Besson \& Rowe [48]

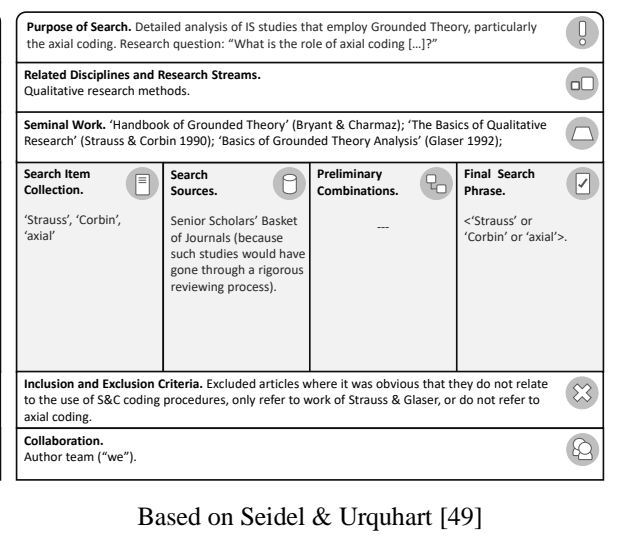

Figure 4. Illustrative scenarios demonstrating the framework's applicability

\section{Discussion and Conclusion}

The sources examined in this study revealed challenges for searching literature and demands in terms of standardized templates that help to document the search strategy in a flexible manner. As an example, although methodological guidance stressed that the entire process has to be documented [5], our analysis of state-of-the-art reviews in IS indicates that this is fulfilled by limited studies: Only 7/51 articles specified a search phrase, there is great heterogeneity in the number of keywords used (between 1-21 terms), and only 28/51 articles followed a systematic procedure. Furthermore, as disclosed through the think alouds, even though the participants were familiar with review procedures, they struggled with the search process, thus asking for "guidance on the derivation of a search phrase". Against this backdrop, we examined the main components of keyword-based searches in IS to design a supporting framework. Therefore, we conceptualized available methodological guidance on literature reviews, literature review-articles, and think aloud-sessions to deduce a set of main review components. Drawing on this conceptualization, we iteratively developed and evaluated the Search Canvas across two major iterations. 
Our contribution is threefold: First, we contribute to an understanding of what are essential components that should be considered and reported during the early stages of a review, and thus help particularly novice researchers to get started with the search. We present a framework for structuring and visualizing search strategies, which importance is emphasized by various authors in IS such as [11] who emphasized that "a search protocol is a useful way to guide and organize the literature search". Although documentation is a major aspect of ensuring transparency (e.g., "quality of a review is rather reflected by the thoroughness of the documentation of the search" [4]), steps for searching and screening are often (still) underrepresented in IS-reviews [5]. Second, since the canvas facilitates exploration and ideation [18, 19], our artifact allows for methodological flexibility $[14,15]$ through refining a search strategy across several iterations, for example, by communicating ideas and collecting feedback from experts. Third, the canvas might help facing challenges in terms of the corpus creation [13] because (a) researchers might pay more attention to critical aspects such as adjacent disciplines and the broader phenomenon's context utilizing the canvas (e.g., as shown during the evaluation) as well as (b) brainstorm on and validate strategy aspects such as search terms and sources through the help of collaboration across disciplines.

Although this study contributes to how to get started with a literature review and how to document the search strategy, it is not free of limitations that open avenues for future research. First, we primarily report descriptive findings from the evaluation and focused on quantitative insights, neglecting the investigation of whether a high amount of keywords leads to ultimately better search phrases. In line with Parè et al. [4], "we must also bring the "quality' element to the discussion of conducting literature reviews" for which reason further steps might be concerned with analyzing 'precision' (i.e., correctness, quality) in addition to 'recall' (i.e., completeness, quantity). Second, even though all reviews require a minimum level in terms of being systematic [14], we did not evaluate the applicability across different review types such as theoretical, narrative, or descriptive reviews [4], especially quantitative-based reviews might demand additional canvas components [5]. Third, whereas a canvas is intended to be the boundary object that is continuously revised within a team of collaborators over a period of time, our evaluation is restricted to a specific timespan and individual use. Further research is required that explores the usage over time, for instance, through a case study or longitudinal study. In doing this, researchers might examine when review teams stop with the actual specification of a search strategy (e.g., adapting concepts such as 'theoretical saturation') to provide guidelines on how to apply the iterative nature of the canvas. Fourth, our study is limited to the group of users, namely IS students. As indicated by the questionnaire (step 5), there seems to be a tendency that more experienced researchers gave higher rankings for the framework's usefulnessmaybe because they did already face several challenges during reviewing the literature. Hence, future endeavors can focus on the target user groups and investigate for which groups the framework is suitable (e.g., IS education). Fifth, referring to the framework's design, the artifact is based on own decisions and interpretations (e.g., in terms of the components and their arrangement) as well as on the underpinning research methods that have been applied, which both have limitations. 
In conclusion, we hope to complement the valuable stream of research on literature review guidance that is already available for the IS discipline as well as shed light on the first steps of deriving and documenting a search strategy. The initial evaluations indicate promising results, especially in supporting novice researchers to perform, specify, and document search strategies with a higher level of transparency.

\section{References}

1. Boell, S.K., Cecez-Kecmanovic, D.: On being 'systematic' in literature reviews in IS. Journal of Information Technology. 30, 161-173 (2015)

2. Levy, Y., Ellis, T.J.: A systems approach to conduct an effective literature review in support of information systems research. Informing Science: International Journal of an Emerging Transdiscipline. 9, 181-212 (2006)

3. Webster, J., Watson, R.T.: Analyzing the past to prepare for the future: Writing a literature review. MIS Quarterly. 26, xiii-xxiii (2002)

4. Paré, G., Trudel, M.-C., Jaana, M., Kitsiou, S.: Synthesizing information systems knowledge: A typology of literature reviews. Information \& Management. 52, 183-199 (2015)

5. Templier, M., Paré, G.: Transparency in literature reviews: An assessment of reporting practices across review types and genres in top IS journals. null. 27, 503-550 (2018)

6. Schryen, G., Benlian, A., Rowe, F., Shirley, G., Larsen, K., Petter, S., Paré, G., Wagner, G., Haag, S., Yasasin, E.: Literature reviews in IS research: What can be learnt from the past and other fields? Communications of the Association for Information Systems. 41, 759-774 (2017)

7. Schryen, G.: Writing qualitative IS literature reviews - Guidelines for synthesis, interpretation and guidance of research. Communications of the Association for Information Systems. 37, 286-325 (2015)

8. Rowe, F.: What literature review is not: Diversity, boundaries and recommendations. European Journal of Information Systems. 23, 241-255 (2014)

9. Thomé, A.M.T., Scavarda, L.F., Scavarda, A.J.: Conducting systematic literature review in operations management. Production Planning \& Control. 27, 408-420 (2016)

10. Cram, W.A., Templier, M., Pare, G.: (Re)considering the concept of literature review reproducibility. Journal of the Association for Information Systems. 21 (2020)

11. vom Brocke, J., Simons, A., Riemer, K., Niehaves, B., Plattfaut, R., Cleven, A.: Standing on the shoulders of giants: Challenges and recommendations of literature search in information systems research. Communications of the Association for Information Systems. 37, 205-224 (2015)

12. Boell, S., Wang, B.: www. litbaskets. io, an IT artifact supporting exploratory literature searches for Information Systems Research. In: Australasian Conference on Information Systems. Perth, Australia (2019)

13. Larsen, K.R., Hovorka, D., Dennis, A., West, J.: Understanding the elephant: The discourse approach to boundary identification and corpus construction for theory review articles. Journal of the Association for Information Systems. 20 (2019)

14. Paré, G., Tate, M., Johnstone, D., Kitsiou, S.: Contextualizing the twin concepts of systematicity and transparency in information systems literature reviews. 25, 493-508 (2016) 
15. Wolfswinkel, J.F., Furtmueller, E., Wilderom, C.P.M.: Using grounded theory as a method for rigorously reviewing literature. European Journal of Information Systems. 22, 45-55 (2013)

16. Schoormann, T., Behrens, D., Fellmann, M., Knackstedt, R.: Sorry, too much information Design principles for supporting rigorous search strategies in literature reviews. In: 20th Conference on Business Informatics, pp. 99-108. IEEE (2018)

17. Boell, S.K., Cecez-Kecmanovic, D.: Debating systematic literature reviews (SLR) and their ramifications for IS: A rejoinder to Mike Chiasson, Briony Oates, Ulrike Schultze, and Richard Watson. Journal of Information Technology. 30, 188-193 (2015)

18. Avdiji, H., Elikan, D., Missonier, S., Pigneur, Y.: A design theory for visual inquiry tools. Journal of the Association for Information Systems. 21 (2020)

19. Strategyzer: The business model canvas: Why and how organizations around the world adopt it. (2015)

20. Osterwalder, A., Pigneur, Y.: Business Model Generation: A handbook for visionaries, game changers, and challengers. John Wiley \& Sons (2010)

21. Gregor, S., Hevner, A.R.: Positioning and presenting design science research for maximum impact. MIS Quarterly. 37, 337-355 (2013)

22. Bandara, W., Furtmueller, E., Gorbacheva, E., Miskon, S., Beekhuyzen, J.: Achieving rigor in literature reviews: Insights from qualitative data analysis and tool-support. Communications of the Association for Information Systems. 37, 154-204 (2015)

23. Tate, M., Furtmueller, E., Evermann, J., Bandara, W.: Introduction to the Special Issue: The literature review in information systems. Communications of the Association for Information Systems. 37, 5 (2015)

24. Boell, S., Cecez-Kecmanovic, D.: A Hermeneutic approach for conducting literature reviews and literature searches. Communications of the Association for Information Systems. 34 (2014)

25. vom Brocke, J., Simons, A., Niehaves, B., Riemer, K., Plattfaut, R., Cleven, A.: Reconstructing the giant: On the importance of rigour in documenting the literature search process. In: 17th European Conference on Information Systems, pp. 2206-2217. Verona, Italy (2009)

26. Okoli, C., Schabram, K.: A guide to conducting a systematic literature review of information systems research. Sprouts: Working Papers on Information System. 10 (2010)

27. Kitchenham, B.: Procedures for performing systematic reviews (2004)

28. Brereton, P., Kitchenham, B.A., Budgen, D., Turner, M., Khalil, M.: Lessons from applying the systematic literature review process within the software engineering domain. Journal of Systems and Software. 80, 571-583 (2007)

29. Kitchenham, B.A., Brereton, O.P., Budgen, D.: Protocol for extending an existing tertiary study of systematic literature reviews in software engineering. Keele University, Keele University (2006)

30. Kitchenham, B.A., Mendes, E., Travassos, G.H.: Protocol for systematic review of withinand cross-company estimation models. Keele University (2008)

31. Booth, A., Sutton, A., Papaioannou, D.: Systematic approaches to a successful literature review. Sage (2016)

32. Täuscher, K., Abdelkafi, N.: Visual tools for business model innovation: Recommendations from a cognitive perspective. Creativity and Innovation Management. 26, 160-174 (2017)

33. John, T., Szopinski, D.: Towards explaining the popularity of the business model canvas: A dual coding approach. In: Multikonferenz Wirtschaftsinformatik. Lüneburg, Germany (2018) 
34. Morana, S., Scheid, M., Gau, M., Benke, I., vom Brocke, J., Fettke, P., Maedche, A.: Research prototype: The design canvas in MyDesignProcess. com. In: International Conference on Design Science Research in Information Systems and Technology. Chennai, India (2018)

35. Kühne, B., Böhmann, T.: Requirements for representing data-driven business models Towards extending the business model canvas. In: 24th Americas Conference on Information Systems. New Orleans, USA (2018).

36. Poeppelbuss, J., Lubarski, A.: Modularity Canvas - A framework for visualizing potentials of Service Modularity. In: Wirtschaftsinformatik (WI). Siegen, Germany (2019)

37. Schoormann, T., Behrens, D., Kolek, E., Knackstedt, R.: Sustainability in business modelsA literature-review-based design-science-oriented research agenda. In: 24th European Conference on Information Systems. Istanbul, Turkey (2016)

38. Star, S.L.: This is Not a Boundary Object: Reflections on the origin of a concept. Science, Technology, \& Human Values. 35, 601-617 (2010)

39. Hevner, A.R., March, S.T., Park, J., Ram, S.: Design science in information systems research. MIS Quarterly. 28, 75-105 (2004)

40. Someren, M. van, Barnard, Y.F., Sandberg, J.A.: The think aloud method: A practical approach to modelling cognitive processes. Londen: Academic Press (1994)

41. Mindel, V., Mathiassen, L.: Contextualist inquiry into IT-enabled hospital revenue cycle management: Bridging research and practice. Journal of the Association for Information Systems. 16, 1016 (2015)

42. Roberts, N., Galluch, P.S., Dinger, M., Grover, V.: Absorptive capacity and information systems research: Review, synthesis, and directions for future research. MIS quarterly. 36 (2012)

43. Ericsson, K.A., Simon, H.A.: Verbal reports as data. Psychological review. 87, 215-251 (1980)

44. Jaspers, M., Steen, T., Bos, C., Geenen, M.: The think aloud method: A guide to user interface design. International Journal of Medical Informatics. 73, 781-795 (2004)

45. Hwang, W., Salvendy, G.: Number of people required for usability evaluation: the $10 \pm 2$ rule. Communications of the ACM. 53, 130 (2010)

46. Peffers, K., Rothenberger, M., Tuunanen, T., Vaezi, R.: Design science research evaluation. In: Design Science Research in Information Systems. Advances in Theory and Practice. pp. 398-410. Springer, Berlin, Heidelberg (2012)

47. Miller, G.A.: The magical number seven, plus or minus two: Some limits on our capacity for processing information. Psychological review. 63, 81 (1956)

48. Besson, P., Rowe, F.: Strategizing information systems-enabled organizational transformation: A transdisciplinary review and new directions. The Journal of Strategic Information Systems. 21, 103-124 (2012)

49. Seidel, S., Urquhart, C.: On emergence and forcing in information systems grounded theory studies: The case of Strauss and Corbin. Journal of Information Technology. 28, 237-260 (2013) 